\title{
Porcine Dermal Xenograft as Augmentation in the Treatment of Large Rotator Cuff Tears: Clinical and Magnetic Resonance Results at 2-Year Follow-Up
}

\author{
Alessandro Castagna ${ }^{1}$ Eugenio Cesari ${ }^{2}$ Berardo Di Matteo ${ }^{3,4}$ Marcello Osimani ${ }^{5}$ Raffaele Garofalo ${ }^{6}$ \\ Elizaveta Kon ${ }^{3,4}$ Maurilio Marcacci ${ }^{3,4}$ Claudio Chillemi ${ }^{7}$
}

${ }^{1}$ Center for Shoulder and Elbow Surgery, Humanitas Clinical and Research Center, Rozzano, Milan, Italy

2 Shoulder Surgery Unit, Humanitas Gavazzeni Institute, Bergamo, Italy

${ }^{3}$ Department of Biomedical Sciences, Humanitas University, Rozzano, Milan, Italy

${ }^{4}$ Humanitas Clinical and Research Center, Rozzano, Milan, Italy

${ }^{5}$ Department of Radiological Sciences, University of Rome

"Sapienza," ICOT, Latina, Italy

6 Upper Limb Unit, F. Miulli Hospital, Acquaviva delle Fonti, Bari, Italy

7 Department of Othopaedics and Traumatology - Istituto Chirurgico Ortopedico Traumatologico (ICOT), Latina, Italy

Joints 2018;6:135-140.

\begin{abstract}
Address for correspondence Berardo Di Matteo, MD, Center for Functional and Biological Reconstruction of the Knee, Humanitas Clinical and Research Center, Via A. Manzoni 113, 20189 Rozzano, Milan, Italy (e-mail: berardo.dimatteo@gmail.com).
\end{abstract}

Purpose The aim of the present retrospective study is to describe the results obtained at 2-year follow-up by using a porcine dermis-derived collagen membrane implanted as augmentation to treat large rotator cuff tears.

Methods Thirty-five patients in total were included according to the following criteria: large or massive rotator cuff tear, confirmed during surgery, measuring between 3 and $5 \mathrm{~cm}$ in width and stage 1 to 2 fatty infiltration documented at magnetic resonance imaging (MRI). Patients underwent arthroscopic repair of the cuff augmented by the implantation of a porcine dermal collagen membrane. Patients were evaluated up to 24 months after surgery by the Constant score and MRI imaging to assess functional outcomes and re-tear rate. The results obtained were compared to those of a matched cohort of 35 patients operated by arthroscopic repair alone by the same surgical team.

Results The application of the porcine membrane proved to be safe without scaffoldrelated adverse events documented. A statistically significant difference in the Constant score in favor of the treatment group was documented at the final evaluation $(p=0.036)$. Furthermore, a subgroup analysis revealed that patients treated by augmentation and presenting re-tear at MRI showed a significantly higher functional outcome compared with control patients with MRI evidence of re-tear $(p=0.0136)$. Conclusion Arthroscopic repair augmented by porcine dermal xenograft for the treatment of chronic and retracted rotator cuff tears with low-grade fatty degeneration proved to be safe and also effective, with higher functional score compared with the arthroscopic repair alone.

Level of Evidence This is a Level III, retrospective cohort study. received

May 16, 2018

accepted

October 20, 2018

published online

December 12, 2018
DOI https://doi.org/

10.1055/s-0038-1676106. ISSN 2282-4324.
Copyright (c) 2018 Georg Thieme Verlag License terms

KG Stuttgart · New York
(요 $\Theta \circledast$ 


\section{Introduction}

The treatment of symptomatic extensive rotator cuff tears, defined as massive and large tears, represents a challenging problem in shoulder surgery. Traditional repair techniques are associated with variable failure rates due to excessive tension on the repair ${ }^{1}$ and to the presence of degenerated tendon tissue, which has poor healing potential. ${ }^{2}$

A high incidence of failure follows repair of large and massive tears, particularly when they are chronic and the tendons show degenerative changes. Failure can happen as a re-tear, but in many of the cases, it is the effect of non-healing. ${ }^{3}$ Patients with failed repair have inferior clinical outcomes compared with those with intact repair. ${ }^{4}$ To overcome the limitations of traditional repair, regenerative medicine techniques have been under investigation in recent years. The aim is to enhance and stimulate the formation of a tendon tissue with histological and mechanical properties resembling those of the native tendon. Based on these premises, many attempts to reconstruct the rotator cuff by using different kinds of grafts have been performed, using both synthetic materials $s^{5,6}$ and biologic tissues. Autograft biceps tendon, ${ }^{7}$ fascia lata, ${ }^{8}$ allograft freeze dried rotator cuff, ${ }^{9}$ and patellar tendon ${ }^{10}$ have been used in the past with variable results. Recently, several tissue-engineered allografts and xenografts have been developed to augment rotator cuff repairs. Such implants should provide a stronger repair in the presence of poor-quality tissue, and in some cases, they act as a bridging scaffold to enhance healing. Most of the research studies are concentrated on obtaining biological scaffolds able to provide an initial mechanical augmentation to the repaired cuff, absorbing part of the stress and thereby protecting the suture. At the same time, the scaffold should be, since the early phases of healing, a ground for host cell colonization, leading to the formation of a tendon structure with similar histological and mechanical features of the native one, but with a greater thickness. Additional requisites for these scaffolds should be a full biocompatibility and tolerability, complete integration at the end of the healing process, high suture retention properties, and ease to handle for arthroscopic application. Cross-linking provides resistance to collagenase enzymes that are responsible for the breakdown and resorption of implanted collagen. ${ }^{11}$

After implantation, the patch should support fibroblast infiltration and revascularization so that it is gradually incorporated into the surrounding tissue, thus providing strength and support while inhibiting wound contraction through scarring. ${ }^{12-14}$

The aim of the present retrospective study is to describe the results obtained at 2-year follow-up by using a porcine dermisderived collagen membrane implanted as augmentation to treat large rotator cuff tears. The results obtained were then compared with those of a matched control group of patients who underwent standard arthroscopic repair alone.

\section{Methods}

The present study was approved by our Institutional Ethic Committee and all patients gave written informed consent to the use of their clinical data for scientific purposes.

\section{Participants}

Thirty-five patients ("treatment group") were included in the study according to the following inclusion criteria: large or massive rotator cuff tear $(3-5 \mathrm{~cm}$ in width) documented by preoperative magnetic resonance imaging (MRI) and confirmed during surgery; pain and functional disability refractory to conservative treatment; stage 1 or 2 fatty infiltration of the supraspinatus muscle on MRI according to the grading system of Goutallier et al modified by Fuchs for MRI ${ }^{15,16}$; integrity of the teres minor and subscapularis tendons; availability of postoperative MRI of the affected shoulder (performed 24 months after surgery); and followup period of 24 months. Exclusion criteria were positive Patte test and/or drop-arm sign at baseline clinical evaluation, previous surgeries or traumas to both shoulders, arthritic and/or septic conditions of both shoulders, diabetes, neuromuscular diseases, smoking, and previous steroid injections into the affected shoulder.

Patients of the treatment group were compared with a control group consisting of a matching cohort of 35 patients operated in the same time interval in our department by standard arthroscopic repair without augmentation. Patients of the control group were selected based on the same inclusion criteria described above and with the same demographic characteristics at baseline evaluation. All the patients of the matching cohort were evaluated at 24-month follow-up for clinical and imaging outcomes similar to those of the treatment group.

\section{Intervention}

The patient was placed in lateral decubitus position and supraclavicular regional block was used for anesthesia. After preparation and draping, a posterior portal was established $1 \mathrm{~cm}$ medial and $2 \mathrm{~cm}$ distal to the posterolateral corner of the acromion. The position of this portal was proximal and lateral to the standard posterior portal. This "higher" position facilitates subacromial work. A working portal was then established in the anterosuperior rotator interval by the use of an "inside-out" technique. Diagnostic arthroscopy was started and associated glenohumeral pathology was addressed including long head of the biceps. The arthroscope was then introduced into the subacromial space. A lateral portal was created at the midpoint between the anterior and posterior aspects of the acromion. A thorough subacromial bursectomy was performed to gain proper visualization and rotator cuff tendons were mobilized by releasing any adhesions, especially within the rotator interval and supraglenoid recess. Cuff mobility was assessed by pulling laterally the retracted supraspinatus tendon and ensuring that its lateral margin could be reduced to the greater tuberosity without significant tension with the arm in neutral abduction and rotation. The tuberosity was prepared with a shaver and burr, while care was taken not to violate the cortex. To reduce tension on the repair, up to 3 to $5 \mathrm{~mm}$ of medialization of the footprint was performed by removing the lateral-most cartilage. Accessory anterolateral and posterolateral portals $3 \mathrm{~cm}$ off the anterolateral and posterolateral corners of the acromion were also performed to aid the procedure. 
In the control group, the rotator cuff was repaired by a single-row technique using two triple loaded suture anchors (Trevo; Linvatec, Largo, Florida, United States) laterally positioned. All sutures were passed through the cuff with a sharp-tipped penetrator and then tied by conventional arthroscopic knot-tying techniques.

In the treatment group, a porcine dermis-derived collagen membrane (Conexa Reconstructive Tissue Matrix, Wright Medical Group N.V., Tennessee, United States) was used to augment the repair. No tear bridging was performed. ${ }^{17}$ The V-sled technique ${ }^{18}$ was adopted to fix both the cuff repair and the scaffold. In this case, two $5.5 \mathrm{~mm}$ triple-loaded bioabsorbable anchors (Trevo, Linvatec) were used. The first anchor was placed anterolaterally (ALA: anterolateral anchor) and the second posterolaterally (PLA: posterolateral anchor). The repair was performed using two high-strength sutures from each anchor. The third suture from each anchor was then retrieved out of the accessories portals used for the insertion of the anchors. Two free high strength sutures were passed through the muscle anteromedially (AM) and posteromedially (PM) in line with the ALA and PLA, respectively, approximately $3 \mathrm{~cm}$ medially from anchors, while the distance between the AM and PM was the same as that between ALA and PLA. Size of the scaffold was established using a measuring probe introduced through the lateral portal. The patch was then cut to cover the entire lesion and, using a free suture needle, the posterior limb of the AM suture (PL-AM) was passed through the medial edge of the patch with an anteroposterior direction moving from top to bottom. The passage was complete when the suture reached the midpoint of the medial edge of the patch. Similarly, the anterior limb of the PM suture (AL-PM) was passed through the medial edge of the patch but this time moving in a posterior-anterior direction. Therefore, the PL-AM and ALPM sutures were tied together over the patch. The third suture from each anchor (ALA and PLA) was then passed, respectively, through the anterolateral and posterolateral corner of the patch. By pulling the posterior limb of the AM suture and the anterior limb of the PM sutures, the patch folded and slided through the lateral portal easily entering the subacromial space. With the use of a probe, the patch was unrolled, recovering its original shape. The free portion of AM and PM sutures was then loaded in a knotless anchor that was placed approximately $1.5 \mathrm{~cm}$ distal to the edge of the repaired cuff. In this way, the two sutures were stretched on the patch, stabilizing, and compressing it with a V-shape bridge. The remaining third suture of each anchor was then tied (-Fig. 1). The arm was brought through passive range of motion (ROM) to ensure appropriate construct tension without gap formation.

Postoperatively, all patients were immobilized in a sling with a $15^{\circ}$ abduction pillow. At 4 weeks, patients began pendulum and passive ROM exercises on the scapular plane. At 6 weeks, sling use was discontinued and patients began active-full assisted ROM exercises. At 8 weeks, patients began active ROM and isometric rotator cuff strengthening, with progression toward return to full activity at 6 months postoperatively.

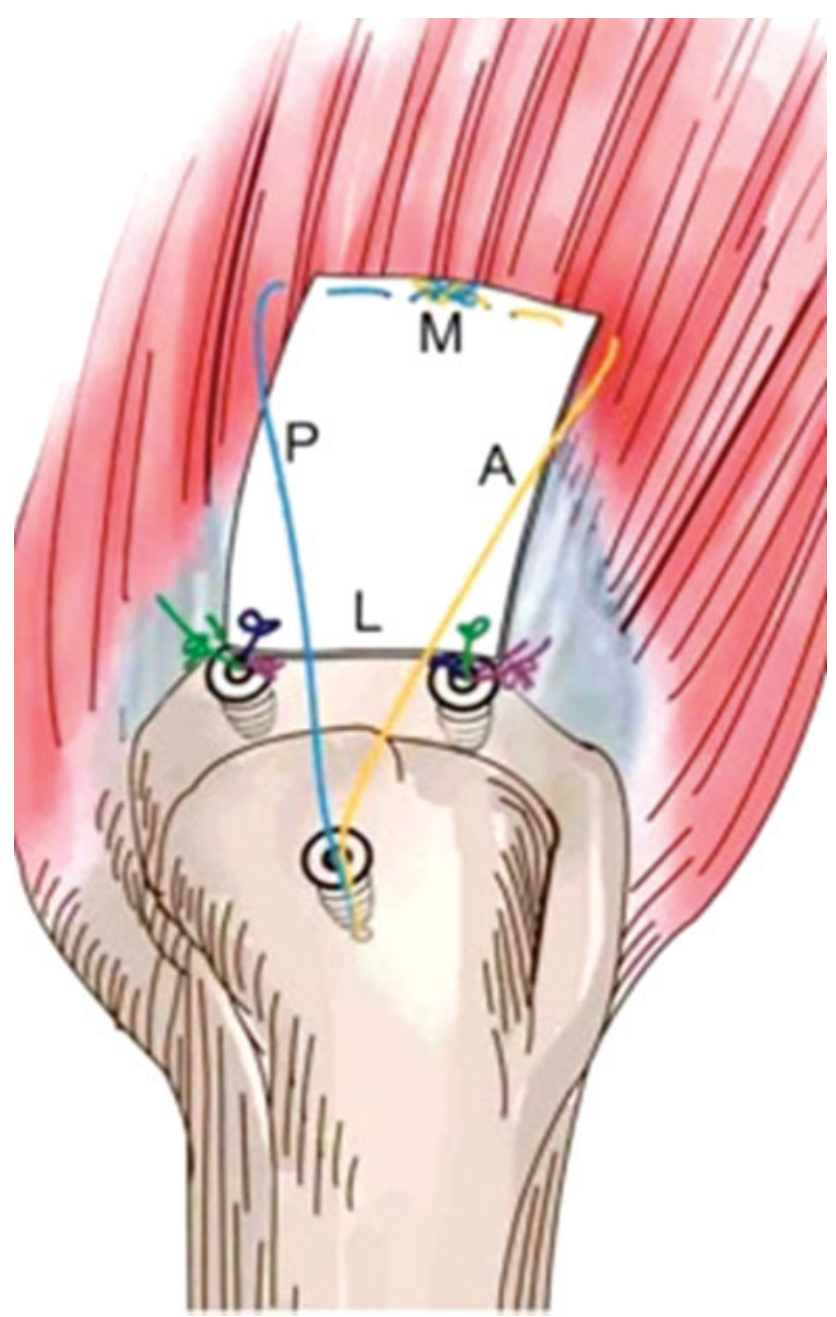

Fig. 1 Arthroscopic implantation technique of the graft.

\section{Outcome Measurements}

Each patient underwent preoperative imaging with radiographs and MRI. Clinical evaluation was performed on the day before surgery and 24 months postoperatively. Functional outcome was assessed by the Constant score. ${ }^{19}$ Active ROM of the shoulder to the point of pain was measured with a goniometer, including forward flexion (FF), external rotation (ER) with the arm at the side, and internal rotation (IR) at the back. A digital dynamometer (micro FET2 MT; Hoggan Health Industries, West Jordan, Utah, United States) was used to quantitatively assess the isometric muscle strength in both the affected and unaffected upper extremity in the seated position. Abduction strength was tested with the arm abducted to $90^{\circ}$ in the scapular plane with the elbow extended and the forearm pronated. The measurement was made three times and the average of the three measurements was obtained and used for strength assessment in the Constant score.

Fatty infiltration of the rotator cuff was evaluated on preoperative MRI. ${ }^{20,21}$ Cuff integrity on MRI was evaluated 24 months postoperatively by a blinded musculoskeletal radiologist. 
MRI was performed with a 1.5-T closed-type scanner (Excelart Vantage powered by Atlas or Visart/EX; Toshiba, Otawara, Japan). Oblique coronal, oblique sagittal, and axial T2-weighted images were acquired for structural and qualitative assessment of the rotator cuff tendons and the graft, and repair integrity was evaluated. The slice thickness was $4 \mathrm{~mm}$, and the interslice gap was $0.5 \mathrm{~mm}$ in the former scanner and $0.8 \mathrm{~mm}$ in the latter scanner. Continuity and re-rupture of the tendon were assessed on MRI scans according to established MRI criteria. ${ }^{20,21}$ A full-thickness re-tear was diagnosed in the presence of a high-signal intensity or when discontinuity of the tendon was observed on one or more of the T2-weighted images.

\section{Statistical Analysis}

All continuous data were expressed as mean and standard deviation; categorical variables were expressed as frequency and percentages. The Wilcoxon nonparametric test was used to test the differences at different follow-up times. The Mann-Whitney nonparametric test was performed to assess between-group differences of continuous data. The Spearman rank correlation was used to assess correlation between rank and continuous data, and the Kendall tau ordinal correlation was used to assess correlation of ordinal data. Fisher's chi-square test was performed to investigate the relationships between dichotomous variables. Pearson's chisquare test evaluated by exact methods for small samples was performed to investigate the relationships between grouping variables. All nonparametric tests were evaluated by exact methods for small samples. For all tests, $p<0.05$ was considered significant. All statistical analysis was performed with SPSS software, version 19.0 (IBM, Armonk, New York, United States).

\section{Results}

There were no significant differences between the two groups in terms of sex, age, follow-up period, dominant hand, and Constant score at baseline.

The application of the porcine membrane proved to be safe and no intraoperative or perioperative complication (including neural injury, wound infection, or suture anchor problems) was registered.

Postoperative MRI at the final 24-month follow-up showed that 7 shoulders in the treatment group (21.9\%) presented retears of the supraspinatus tendon, whereas $13(33.3 \%)$ occurred in the control group. No significant difference was documented between groups in terms of re-tear rate.

With respect to preoperative value, the mean Constant score was significantly higher at 24-month follow-up in both groups $(p<0.05)$. In particular, a statistically significant difference, in favor of the treatment group was documented at the final evaluation $(p=0.036)$. Furthermore, a subgroup analysis revealed that patients treated by augmentation and presenting re-tear at MRI showed a significantly higher Constant score compared with patients of the control group with MRI evidence of re-tear $(p=0.0136)$. In the treatment group, no significant difference was detected at 24 months among
Table 1 Mean Constant score in the treatment and control groups (overall results and subgroup analysis according to MRI evidence of re-tear)

\begin{tabular}{|l|l|l|l|}
\hline Constant score & $\begin{array}{l}\text { Treatment } \\
\text { group } \\
\text { (mean } \pm \text { SD) }\end{array}$ & $\begin{array}{l}\text { Control group } \\
\text { (mean } \pm \text { SD) }\end{array}$ & $p$-Value \\
\hline Preoperative & $37.2+8.2$ & $37.3+5.3$ & n.s. \\
\hline $\begin{array}{l}\text { Follow-up } \\
\text { (24 months) }\end{array}$ & $71.4+11.8$ & $63.9+11.9$ & 0.036 \\
\hline $\begin{array}{l}\text { Follow-up } \\
\text { (24 months) } \\
\text { Patients with } \\
\text { MRI evidence } \\
\text { of re-tear }\end{array}$ & $65.3+10.8$ & $53.4+9.7$ & 0.0136 \\
\hline
\end{tabular}

Abbreviations: MRI, magnetic resonance imaging; SD, standard deviation; n.s., non significant.

patients with and without re-tear at MRI evaluation. Conversely, in the control group, there was a significant clinical difference between patients with fully healed supraspinatus tendon and those with evidence of re-injury $(63.9 \pm 11.9$ vs. $53.4 \pm$ 9.7, respectively; $p<0.001)(-$ Table 1$)$.

\section{Discussion}

The results of the present study suggest that arthroscopic repair augmented with a porcine dermal collagen scaffold might represent an effective method in the treatment of repairable large rotator cuff tears. There were no complications directly related to the use of the porcine dermal graft, and in particular, there was no evidence of tissue rejection. A significantly higher Constant score was observed in the treatment group 24 months after surgery compared with the matched control group, and patients who received the augmentation presented higher clinical scores even in case of re-tear. Interestingly, the augmentation by porcine graft prevented the deterioration of clinical scores in case of re-tear, and, in the treatment group, we reported no clinical difference among patients with or without evidence of re-tear. However, the lack of significant difference in re-tear rate between treatment and control group might suggest that the patch itself is not able to restore a full integrity of the tendon, thus meaning that a complete regeneration cannot be achieved.

Nonetheless, a recent systematic review reported that, despite patients with intact repairs had significantly higher strength in forward elevation and ER, these objective differences are not truly clinically relevant for the final outcome and overall patients' satisfaction. ${ }^{22}$ Although our results could not endorse the hypothesis that the patch augmentation significantly improved the healing rate of the rotator cuff, the superior clinical outcome reported at 24 months and the higher scores in patients with MRI signs of re-tear suggest a beneficial contribution of the porcine membrane that should be evaluated at longer follow-up. Perhaps, despite the number of patients included in the present study allowed to detect a significant difference in functional outcome (which was the primary end-point of the study), a higher number would have 
been required to assess also a significant difference in terms of re-tear rate at MRI evaluation. In particular, in the group of patients with re-tear, we support the idea that the scaffold, even in absence of an anatomic and complete healing of tendons, could partially restore the superior stability of the glenohumeral joint. As previously demonstrated by Mihata et al, ${ }^{23}$ patients with massive rotator cuff tear have a defect of the superior capsule, which is located on the inferior surface of the supraspinatus and infraspinatus tendons. Such defect significantly increases translation of the humeral head, causing a reduction in the acromion-humeral distance and thus resulting in subacromial impingement. To restore the superior stability of glenohumeral joint in massive tear, in the last years, a new procedure called superior capsular reconstruction has been popularized: a graft is attached medially to the superior glenoid and laterally to the greater tuberosity. In our study, we believe that, even in patients with a recurrence of rotator cuff tear, the patch, which is attached medially to the myotendinous junction and laterally to the greater tuberosity, could partially act as a superior stabilizer for the glenohumeral joint and could then be responsible for the functional and clinical improvement observed in these patients compared with controls.

The results of our study are in line with the findings of previous studies reporting the use of patch grafts to reinforce rotator cuff repair. ${ }^{24}$ In clinical practice, several different grafts have been tested, with variable outcomes. Recent studies showed positive outcomes when anatomic ${ }^{25}$ or partial $^{26}$ repairs were augmented by using patches derived from porcine dermis. Also, open reconstruction of irreparable massive or full-thickness two-tendon rotator cuff tears by dermal tissue matrix xenograft showed improvement in pain, ROM, strength, and subjective functional outcomes. ${ }^{27}$ Recently, a study demonstrated superior outcome in favor of polypropylene augmentation versus collagen patch for postero-superior massive rotator cuff tear. ${ }^{28}$

Several studies reported that biological augmentation with acellular human dermal matrix allografts had successful clinical outcomes. ${ }^{29-31}$ Barber et $\mathrm{al}^{32}$ reported that, among rotator cuff repairs that underwent such biological augmentation, $85 \%$ remained intact compared with $40 \%$ of controls. Conversely, other authors demonstrated unfavorable re-tear rates and clinical outcome scores with the use of a porcine small intestine submucosa (SIS) graft. A clinical study showed very poor results, with $91 \%$ re-tear after augmentation with porcine SIS of large and massive rotator cuff tears. ${ }^{20}$ This was further confirmed by other studies showing similar modest outcomes. ${ }^{33,34}$ Interestingly, failure was attributed to rapid resorption of the SIS patch and insufficient mechanical support of the repaired tear. ${ }^{33}$ Based on these findings from the available literature, in our series we used a porcine dermal membrane for augmentation because such xenograft does not induce an immune response and has structural and biomechanical properties similar to those of native rotator cuff tendons. ${ }^{25}$

The biological aspects of tendon healing play a significant role in the ultimate clinical outcomes. ${ }^{31,35}$ In vitro mechanical tests have since demonstrated that dermal-based xenografts are not only stronger than mucosal products but also less likely to fail due to suture pullout. ${ }^{32}$ Nevertheless, a sheep model comparing a porcine dermis patch to the porcine SIS has shown superior performance of the dermis-based implant and various problems with the porcine SIS, namely an elevation of the inflammation plasma marker fibrinogen and ossification. Some authors observed that porcine SIS degraded faster than dermis and is almost completely absorbed within 9 weeks. ${ }^{36}$

The mechanical advantages of porcine dermal collagen patch over porcine SIS, which are related to the presence of collagen cross-linking, provide an explanation for the better results obtained in the present study. Furthermore, as shown by the absence of adverse clinical reactions in this series, porcine dermal collagen graft is no allergenic, nontoxic, and does not elicit a foreign body response. It is, therefore, less likely to fail in the early postoperative period. This is in contrast to porcine SIS, which has been shown to elicit inflammatory reactions requiring open debridement in up to $16 \%$ of rotator cuff repairs. ${ }^{37}$

Some limitations of the present study must be acknowledged. First, the study design is retrospective with a matched control group, whereas the ideal setting would have been a rotator cuff tear. Second, the short-term followup did not allow to establish if biologic augmentation is able to provide stable superior results over time. Third, only tendons with stage 1 to 2 of fatty infiltration were included, so further studies are needed to evaluate massive cuff rupture involving two or more tendons with fatty infiltration over stage 2.

In conclusion, arthroscopic repair augmented by porcine dermal xenograft for the treatment of chronic and retracted rotator cuff tears with low-grade fatty degeneration (Goutallier stages 1-2) proved to be safe and also showed positive clinical outcome, with higher functional scores compared with the arthroscopic repair alone. In particular, even patients with MRI evidence of re-tear showed significantly higher functional score after augmentation with the scaffold.

Note

All the data, due to privacy reasons, will be available upon motivated request to the corresponding author of the present article.

\section{Funding}

None.

Conflict of Interest

None declared.

Acknowledgment

The authors would like to thank Martina Arcidiacono, MD (Humanitas Clinical and Research Institute), for her help in manuscript formatting and checking.

\section{References}

1 Davidson PA, Rivenburgh DW. Rotator cuff repair tension as a determinant of functional outcome. J Shoulder Elbow Surg 2000;9 (06):502-506 
2 Matthews TJ, Hand GC, Rees JL, Athanasou NA, Carr AJ. Pathology of the torn rotator cuff tendon. Reduction in potential for repair as tear size increases. J Bone Joint Surg Br 2006;88(04):489-495

3 Chillemi C, Petrozza V, Garro L, et al. Rotator cuff re-tear or nonhealing: histopathological aspects and predictive factors. Knee Surg Sports Traumatol Arthrosc 2011;19(09):1588-1596

4 Harryman DT II, Mack LA, Wang KY, Jackins SE, Richardson ML, Matsen FA III. Repairs of the rotator cuff. Correlation of functional results with integrity of the cuff. J Bone Joint Surg Am 1991;73 (07):982-989

5 Mura N, O'Driscoll SW, Zobitz ME, Heers G, An KN. Biomechanical effect of patch graft for large rotator cuff tears: a cadaver study. Clin Orthop Relat Res 2003;(415):131-138

6 Ozaki J, Fujimoto S, Masuhara K, Tamai S, Yoshimoto S. Reconstruction of chronic massive rotator cuff tears with synthetic materials. Clin Orthop Relat Res 1986;(202):173-183

7 Neviaser JS. Ruptures of the rotator cuff of the shoulder. New concepts in the diagnosis and operative treatment of chronic ruptures. Arch Surg 1971;102(05):483-485

8 Baker AR, McCarron JA, Tan CD, Iannotti JP, Derwin KA. Does augmentation with a reinforced fascia patch improve rotator cuff repair outcomes? Clin Orthop Relat Res 2012;470(09):2513-2521

9 Neviaser JS, Neviaser RJ, Neviaser TJ. The repair of chronic massive ruptures of the rotator cuff of the shoulder by use of a freeze-dried rotator cuff. J Bone Joint Surg Am 1978;60(05):681-684

10 Moore DR, Cain EL, Schwartz ML, Clancy WG Jr. Allograft reconstruction for massive, irreparable rotator cuff tears. Am J Sports Med 2006;34(03):392-396

11 Jarman-Smith ML, Bodamyali T, Stevens C, Howell JA, Horrocks M, Chaudhuri JB. Porcine collagen crosslinking, degradation and its capability for fibroblast adhesion and proliferation. J Mater Sci Mater Med 2004;15(08):925-932

12 Adedeji OA, Bailey CA, Varma JS. Porcine dermal collagen graft in abdominal-wall reconstruction. Br J Plast Surg 2002;55(01):85-86

13 Barrington JW, Dyer R, Bano F. Bladder augmentation using Pelvicol implant for intractable overactive bladder syndrome. Int Urogynecol J Pelvic Floor Dysfunct 2006;17(01):50-53

14 Liyanage SH, Purohit GS, Frye JN, et al. Anterior abdominal wall reconstruction with a Permacol implant. J Plast Reconstr Aesthet Surg 2006;59(05):553-555

15 Goutallier D, Postel JM, Gleyze P, Leguilloux P, Van Driessche S. Influence of cuff muscle fatty degeneration on anatomic and functional outcomes after simple suture of full-thickness tears. J Shoulder Elbow Surg 2003;12(06):550-554

16 Fuchs B, Weishaupt D, Zanetti M, Hodler J, Gerber C. Fatty degeneration of the muscles of the rotator cuff: assessment by computed tomography versus magnetic resonance imaging. J Shoulder Elbow Surg 1999;8(06):599-605

17 Dejardin LM, Arnoczky SP, Ewers BJ, Haut RC, Clarke RB. Tissueengineered rotator cuff tendon using porcine small intestine submucosa. Histologic and mechanical evaluation in dogs. Am J Sports Med 2001;29(02):175-184

18 Chillemi C, El Boustany S, Dei Giudici L, Ippolito G. Arthroscopic rotator cuff repair with augmentation: the $\mathrm{V}$-sled technique. Orthop Surg 2012;4(02):109-113

19 Kukkonen J, Kauko T, Vahlberg T, Joukainen A, Aärimaa V. Investigating minimal clinically important difference for Constant score in patients undergoing rotator cuff surgery. J Shoulder Elbow Surg 2013;22(12):1650-1655

20 Sclamberg SG, Tibone JE, Itamura JM, Kasraeian S. Six-month magnetic resonance imaging follow-up of large and massive rotator cuff repairs reinforced with porcine small intestinal submucosa. J Shoulder Elbow Surg 2004;13(05):538-541

21 Zalavras CG, Gardocki R, Huang E, Stevanovic M, Hedman T, Tibone J. Reconstruction of large rotator cuff tendon defects with porcine small intestinal submucosa in an animal model. J Shoulder Elbow Surg 2006;15(02):224-231

22 Russell RD, Knight JR, Mulligan E, Khazzam MS. Structural integrity after rotator cuff repair does not correlate with patient function and pain: a meta-analysis. J Bone Joint Surg Am 2014; 96(04):265-271

23 Mihata T, McGarry MH, Pirolo JM, Kinoshita M, Lee TQ. Superior capsule reconstruction to restore superior stability in irreparable rotator cuff tears: a biomechanical cadaveric study. Am J Sports Med 2012;40(10):2248-2255

24 Nho SJ, Delos D, Yadav H, et al. Biomechanical and biologic augmentation for the treatment of massive rotator cuff tears. Am J Sports Med 2010;38(03):619-629

25 Badhe SP, Lawrence TM, Smith FD, Lunn PG. An assessment of porcine dermal xenograft as an augmentation graft in the treatment of extensive rotator cuff tears. J Shoulder Elbow Surg 2008; 17(1, Suppl):35S-39S

26 Mori D, Funakoshi N, Yamashita F. Arthroscopic surgery of irreparable large or massive rotator cuff tears with low-grade fatty degeneration of the infraspinatus: patch autograft procedure versus partial repair procedure. Arthroscopy 2013;29(12): 1911-1921

27 Gupta AK, Hug K, Boggess B, Gavigan M, Toth AP. Massive or 2-tendon rotator cuff tears in active patients with minimal glenohumeral arthritis: clinical and radiographic outcomes of reconstruction using dermal tissue matrix xenograft. Am J Sports Med 2013;41(04): 872-879

28 Ciampi P, Scotti C, Nonis A, et al. The benefit of synthetic versus biological patch augmentation in the repair of posterosuperior massive rotator cuff tears: a 3-year follow-up study. Am J Sports Med 2014;42(05):1169-1175

29 Barber FA, Burns JP, Deutsch A, Labbé MR, Litchfield RB. A prospective, randomized evaluation of acellular human dermal matrix augmentation for arthroscopic rotator cuff repair. Arthroscopy 2012;28(01):8-15

30 Bond JL, Dopirak RM, Higgins J, Burns J, Snyder SJ. Arthroscopic replacement of massive, irreparable rotator cuff tears using a GraftJacket allograft: technique and preliminary results. Arthroscopy 2008;24(04):403-409

31 Derwin KA, Baker AR, Spragg RK, Leigh DR, Iannotti JP. Commercial extracellular matrix scaffolds for rotator cuff tendon repair. Biomechanical, biochemical, and cellular properties. J Bone Joint Surg Am 2006;88(12):2665-2672

32 Barber FA, Herbert MA, Coons DA. Tendon augmentation grafts: biomechanical failure loads and failure patterns. Arthroscopy 2006;22(05):534-538

33 Iannotti JP, Codsi MJ, Kwon YW, Derwin K, Ciccone J, Brems JJ. Porcine small intestine submucosa augmentation of surgical repair of chronic two-tendon rotator cuff tears. A randomized, controlled trial. J Bone Joint Surg Am 2006;88(06):1238-1244

34 Phipatanakul WP, Petersen SA. Porcine small intestine submucosa xenograft augmentation in repair of massive rotator cuff tears. Am J Orthop 2009;38(11):572-575

35 Shea KP, McCarthy MB, Ledgard F, Arciero C, Chowaniec D, Mazzocca AD. Human tendon cell response to 7 commercially available extracellular matrix materials: an in vitro study. Arthroscopy 2010;26(09):1181-1188

36 Nicholson GP, Breur GJ, Van Sickle D, Yao JQ Kim J, Blanchard CR. Evaluation of a cross-linked acellular porcine dermal patch for rotator cuff repair augmentation in an ovine model. J Shoulder Elbow Surg 2007;16(5, Suppl):S184-S190

37 Malcarney HL, Bonar F, Murrell GA. Early inflammatory reaction after rotator cuff repair with a porcine small intestine submucosal implant: a report of 4 cases. Am J Sports Med 2005;33(06): 907-911 